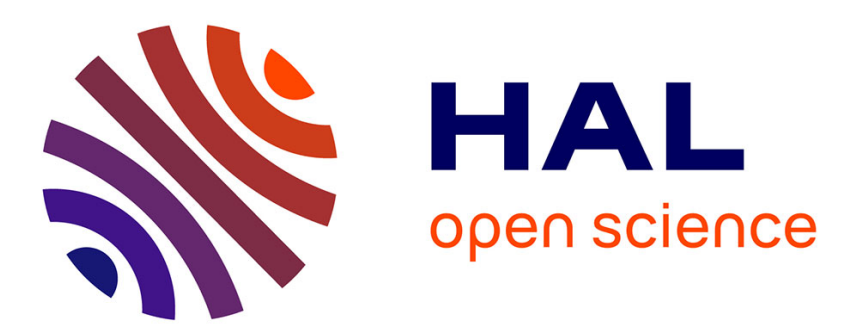

\title{
A COMPARISON OF METHODS FOR SELECTING PREFERRED SOLUTIONS IN MULTIOBJECTIVE DECISION MAKING
}

Enrico Zio, R. Bazzo

\section{- To cite this version:}

Enrico Zio, R. Bazzo. A COMPARISON OF METHODS FOR SELECTING PREFERRED SOLUTIONS IN MULTIOBJECTIVE DECISION MAKING. Cengiz Kahraman. Computational Intelligence Systems in Industrial Engineering, Springer, pp.23-43, 2012, Atlantis Computational Intelligence Systems, Vol. 6. hal-00771555

HAL Id: hal-00771555

https://hal-centralesupelec.archives-ouvertes.fr/hal-00771555

Submitted on 8 Jan 2013

HAL is a multi-disciplinary open access archive for the deposit and dissemination of scientific research documents, whether they are published or not. The documents may come from teaching and research institutions in France or abroad, or from public or private research centers.
L'archive ouverte pluridisciplinaire HAL, est destinée au dépôt et à la diffusion de documents scientifiques de niveau recherche, publiés ou non, émanant des établissements d'enseignement et de recherche français ou étrangers, des laboratoires publics ou privés. 


\title{
CHAPTER 1
}

\section{A COMPARISON OF METHODS FOR SELECTING PREFERRED SOLUTIONS IN MULTIOBJECTIVE DECISION MAKING}

\begin{abstract}
In multiobjective optimization problems, the identified Pareto Frontiers and Sets often contain too many solutions, which make it difficult for the decision maker to select a preferred alternative. To facilitate the selection task, decision making support tools can be used in different instances of the multiobjective optimization search to introduce preferences on the objectives or to give a condensed representation of the solutions on the Pareto Frontier, so as to offer to the decision maker a manageable picture of the solution alternatives.

This paper presents a comparison of some a priori and a posteriori decision making support methods, aimed at aiding the decision maker in the selection of the preferred solutions. The considered methods are compared with respect to their application to a case study concerning the optimization of the test intervals of the components of a safety system of a nuclear power plant. The engine for the multiobjective optimization search is based on genetic algorithms.
\end{abstract}

\section{Introduction}

Multiobjective optimization is central for many reliability and risk analyses in support to the design, operation, maintenance and regulation of complex systems like nuclear power plants. The solutions sought must be optimal with respect to several objectives, generally conflicting: then, one cannot identify a unique, optimal solution satisfying all objectives, but rather a set of possible solutions can be identified where none is best for all objectives. This set of solutions in the space of the decision variables is called the Pareto Set; the corresponding values of the objectives form the Pareto Frontier.

At the end of a multiobjective optimization, the decision maker (DM) has to select the preferred solutions from the Pareto Frontier and Set; this can be a difficult task for large Pareto Frontiers and Sets. For this reason, decision making support tools are developed to aid the DM in selecting the preferred solutions.

There are different approaches for introducing DM preferences in the optimization process, like the ones presented by ATKOSoft, ${ }^{1}$ Rachmawati and Srinivasan ${ }^{14}$ and Coello Coello $;{ }^{6}$ a common classification is based on when the DM is consulted: a priori, a posteriori, or interactively during the search. 
In this work, a comparison of some a priori and a posteriori methods is performed, aimed at characterizing the different approaches in terms of their advantages and limitations with respect to the support they provide to the DM in the preferential solution selection process; to this purpose, not just the quality of the results, but also the possible difficulties of the DM in applying the procedures are considered. In order to base the comparison on solid experience, the methods considered have been chosen among some of those most extensively researched by the authors.

The a priori method considered is the Guided Multi-Objective Genetic Algorithm (G-MOGA) by Zio, Baraldi and Pedroni, ${ }^{18}$ in which the DM preferences are implemented in a genetic algorithm to bias the search of the Pareto optimal solutions.

The first a posteriori method considered has been introduced by the authors ${ }^{20}$ and uses subtractive clustering ${ }^{5}$ to group the Pareto solutions in homogeneous families; the selection of the most representative solution within each cluster is performed by the analysis of Level Diagrams ${ }^{2}$ or by fuzzy preference assignment, ${ }^{19}$ depending on the decision situation, i.e., depending on the presence or not of defined DM preferences on the objectives. The second procedure, is taken from literature ${ }^{11}$ and is a two-step procedure which exploits a Self Organizing Map $(\mathrm{SOM})^{8}$ and Data Envelopment Analysis (DEA $)^{7} 7$ to first cluster the Pareto Frontier solutions and then remove the least efficient

ones. This procedure is here only synthetically described and critically considered with respect to the feasibility of its application in practice.

Instead, the a priori G-MOGA algorithm and the first a posteriori procedure introduced by the authors in 20 , are compared with respect to a case study of literature regarding the optimization of the test intervals of the components of a nuclear power plant safety system; the optimization considers three objectives: system availability to be maximized, cost (from operation \& maintenance and safety issues) and workers exposure time to be minimized. ${ }^{9}$ The a posteriori procedure of analysis is applied to the Pareto Frontier and Set obtained by a standard Multiobjective Genetic Algorithm. ${ }^{9}$

The remainder of the paper is organized as follows: Section 1.0 presents the case study to describe upfront the setting of the typical multiobjective optimization problem of interest; Section 1.Error! Reference source not found. contains the analysis of the different decision making support methods considered; finally some conclusions are drawn in Section 1.Error! Reference source not found. 


\section{Optimization of the test intervals of the components of a nuclear power plant safety system}

The case study here considered is taken from Giuggioli Busacca, Marseguerra and $\mathrm{Zio}^{9}$ and regards the optimization of the test intervals (TIs) of the high pressure injection system (HPIS) of a pressurized water reactor (PWR), with respect to three objectives: mean system availability to be maximized, cost and workers time of exposure to radiation to be minimized. For reader's convenience, the description of the system and of the optimization problem is here reported, as taken from the original literature source with only minor modifications.

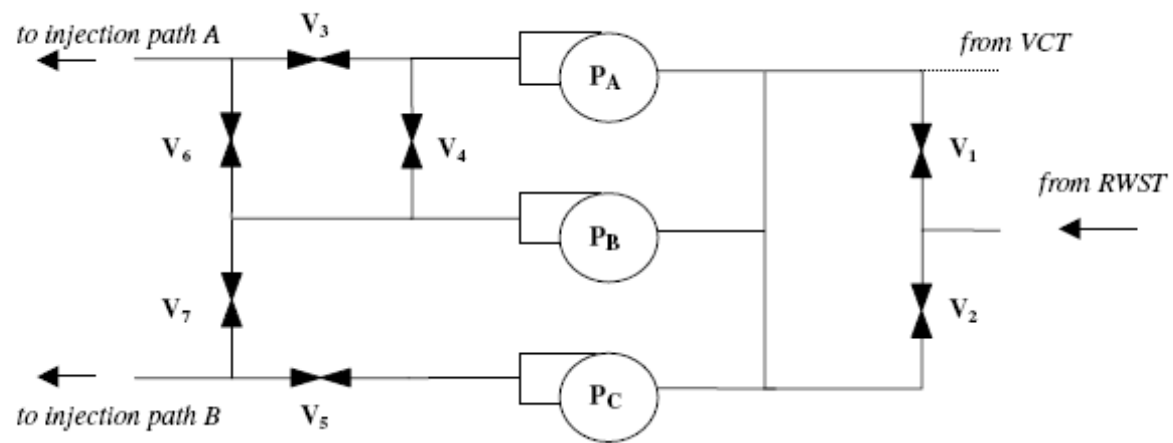

Figure 1: The simplified HPIS system $(\text { RWST }=\text { radioactive waste storage tank })^{9}$

Error! Reference source not found. shows a simplified schematics of a specific HPIS design. The system consists of three pumps and seven valves, for a total of $N_{c}=10$ components. During normal reactor operation, one of the three charging pumps draws water from the volume control tank (VCT) in order to maintain the normal level of water in the primary reactor cooling system (RCS) and to provide a small high-pressure flow to the seals of the RCS pumps. Following a small loss of coolant accident (LOCA), the HPIS is required to supply a high pressure flow to the RCS. Moreover, the HPIS can be used to remove heat from the reactor core if the steam generators were completely unavailable. Under normal conditions, the HPIS function is performed by injection through the valves $V_{3}$ and $V_{5}$ but, for redundancy, crossover valves $V_{4}$ , $V_{5}$ and $V_{7}$ provide alternative flow paths if some failure were to occur in one of the nominal paths. This stand-by safety system has to be inspected periodically to test its availability. A TI of $2190 \mathrm{~h}$ is specified by the technical specifications (TSs) for both the pumps and the valves. However, there are several restrictions 
on the maintenance procedures described in the TS, depending on reactor operations.

For this study, the following assumptions are made:

(1) At least one of the flow paths must be open at all times.

(2) If the component is found failed during surveillance and testing, it is returned to an as-good-as-new condition through corrective maintenance or replacement.

(3) If the component is found to be operable during surveillance and testing, it is returned to an as-good-as-new condition through restorative maintenance.

(4) The process of test and testing requires a finite time; while the corrective maintenance (or replacement) requires an additional finite time, the restorative maintenance is supposed to be instantaneous.

The $N_{c}$ system components are characterized by their failure rate $\lambda_{h}$, $h=1, \ldots ., N_{c}$, the cost of the yearly test $C_{h t, h}$ and corrective maintenance $C_{h c, h}$, the mean downtime due to corrective maintenance $d_{h}$, the mean downtime due to testing $t_{h}$ and their failure on demand probability $\rho_{h}$ (Table 1 ). They are also divided in three groups characterized by different test strategies with respect to the TI $\tau_{h}$ between two successive tests, $h=1, \ldots ., N_{c}, N_{c}=10$; all the components belonging to a same group undergo testing with the same periodicity $T^{g}$, with $g=1,2,3$, i.e., they all have the same test interval $\left(\tau_{h}=T^{g}, \forall\right.$ component $h$ in test group $g$ ).

\begin{tabular}{|l|l|l|l|l|l|l|l|l|}
\hline $\begin{array}{l}\text { Component } \\
\left(\begin{array}{l}j \\
)\end{array}\right.\end{array}$ & $\begin{array}{l}\text { Component } \\
\text { symbol } \\
\text { (Error! } \\
\text { Reference } \\
\text { source not } \\
\text { found.) }\end{array}$ & $\begin{array}{l}\lambda_{h} \\
\left(\mathrm{~h}^{-1}\right)\end{array}$ & $\begin{array}{l}C_{h t, h} \\
(\$ / \mathrm{h})\end{array}$ & $\begin{array}{l}C_{h c, h} \\
(\$ / \mathrm{h})\end{array}$ & $\begin{array}{l}d_{h} \\
(\mathrm{~h})\end{array}$ & $\begin{array}{l}t_{h} \\
(\mathrm{~h})\end{array}$ & $\rho_{h}$ & $g$ \\
\hline 1 & $V_{1}$ & $5.83 \cdot 10^{-6}$ & 20 & 15 & 2.6 & 0.75 & $1.82 \cdot 10^{-4}$ & 1 \\
\hline 2 & $V_{2}$ & $5.83 \cdot 10^{-6}$ & 20 & 15 & 2.6 & 0.75 & $1.82 \cdot 10^{-4}$ & 1 \\
\hline 3 & $V_{3}$ & $5.83 \cdot 10^{-6}$ & 20 & 15 & 2.6 & 0.75 & $1.82 \cdot 10^{-4}$ & 2 \\
\hline 4 & $V_{4}$ & $5.83 \cdot 10^{-6}$ & 20 & 15 & 2.6 & 0.75 & $1.82 \cdot 10^{-4}$ & 3 \\
\hline 5 & $V_{5}$ & $5.83 \cdot 10^{-6}$ & 20 & 15 & 2.6 & 0.75 & $1.82 \cdot 10^{-4}$ & 2 \\
\hline 6 & $V_{6}$ & $5.83 \cdot 10^{-6}$ & 20 & 15 & 2.6 & 0.75 & $1.82 \cdot 10^{-4}$ & 3 \\
\hline 7 & $V_{7}$ & $5.83 \cdot 10^{-6}$ & 20 & 15 & 2.6 & 0.75 & $1.82 \cdot 10^{-4}$ & 3 \\
\hline
\end{tabular}




\begin{tabular}{|l|l|l|l|l|l|l|l|l|}
\hline 8 & $P_{A}$ & $3.89 \cdot 10^{-6}$ & 20 & 15 & 24 & 4 & $5.3 \cdot 10^{-4}$ & 2 \\
\hline 9 & $P_{B}$ & $3.89 \cdot 10^{-6}$ & 20 & 15 & 24 & 4 & $5.3 \cdot 10^{-4}$ & 2 \\
\hline 10 & $P_{C}$ & $3.89 \cdot 10^{-6}$ & 20 & 15 & 24 & 4 & $5.3 \cdot 10^{-4}$ & 2 \\
\hline
\end{tabular}

Any solution to the optimization problem can be encoded using the following array $\theta$ of decision variables:

$\theta=\left\lfloor\begin{array}{lll}T^{1} & T^{2} & T^{3}\end{array}\right]$

Assuming a mission time (TM) of one year $(8760 \mathrm{~h})$, the range of variability of the three TIs is $[1,8760] \mathrm{h}$.

The search for the optimal test intervals is driven by the following three objective functions $J_{i}(\theta), i=1,2,3$ :

Mean Availability, $1-U_{H P I S}$ :

$\max _{\theta} J_{1}(\theta)=\max _{\theta}\left[\left(1-\sum_{v=1}^{N_{M C S}} \prod_{h=1}^{n_{v}} u_{h}^{-v}(\theta)\right)\right]$

Cost, $C: \quad \min _{\theta} J_{2}(\theta)=\min _{\theta}^{v=1}\left[C_{\text {accident }}(\theta)+\sum_{h=1}^{N_{c}} C_{S \& M, h}(\theta)\right]$

Exposure Time, ET: $\min _{\theta} J_{3}(\theta)=\min _{\theta}\left[\sum_{h=1}^{N_{c}} E T_{h}(\theta)\right]$.

For every solution alternative $\theta$ :

the HPIS mean unavailability $\bar{U}_{\text {HPIS }}(\theta)$ is computed from the fault tree for the top event "no flow out of both injection paths A and B"; the boolean reduction of the corresponding structure function allows determining the $N_{M C S}$ system minimal cut sets (MCS); then, the system mean unavailability is expressed as in the argument of the maximization (2), where $n_{v}$ is the number of basic events in the $v-t h$ minimal cut set and $\bar{u}_{h}$ is the mean unavailability of the $h$-th component contained in the $v$-th MCS, $h=1, \ldots, n_{v}:^{12}$

$\bar{u}_{h}^{v}=\rho_{h}+\frac{1}{2} \lambda_{h} \tau_{h}+\left(\rho_{h}+\lambda_{h} \tau_{h}\right) \frac{d_{h}}{\tau_{h}}+\frac{t_{h}}{\tau_{h}}+\gamma_{0}$

where $\gamma_{0}$ is the probability of human error. The simple expression in (5) is valid for $\rho_{h}<0.1$ and $\lambda_{h} \tau_{h}<0.1$, which are reasonable assumptions when considering safety systems.

the cost objective $C(\theta)$ is made up of two major contributions: $C_{S \& M}(\theta)$, the cost associated with the operation of surveillance and maintenance (S\&M) and $C_{\text {accident }}(\theta)$, the cost associated with consequences of accidents possibly occurring at the plant. 
For a given component $h$, the S\&M cost is computed on the basis of the yearly test and corrective maintenance costs. For a giyen mission time, TM, the number of tests performed on component $h$ are $\frac{19}{3}$; of these, on average, a fraction equal to $\left(\rho_{h}+\lambda_{h} \tau_{h}\right)$ demands also a corrétive maintenance action of duration $d_{h}$; thus, the $\mathrm{S} \& \mathrm{M}$ costs amount to:

$C_{S \& M, h}(\theta)=C_{h t, h} \frac{T M}{\tau_{h}} t_{h}+C_{h c, h}\left(\rho_{h}+\lambda_{h} \tau_{h}\right) \frac{T M}{\tau_{h}} d_{h}, h=1, \ldots, N_{c}$

Concerning the accident cost contribution, it is intended to measure the costs associated to damages of accidents which are not mitigated due to the HPIS failing to intervene. A proper analysis of such costs implies accounting for the probability of the corresponding accident sequences; for simplicity, but with no loss of generality, consideration is here limited only to the accident sequences relative to a small LOCA event tree ${ }^{17}$ (Error! Reference source not found.).

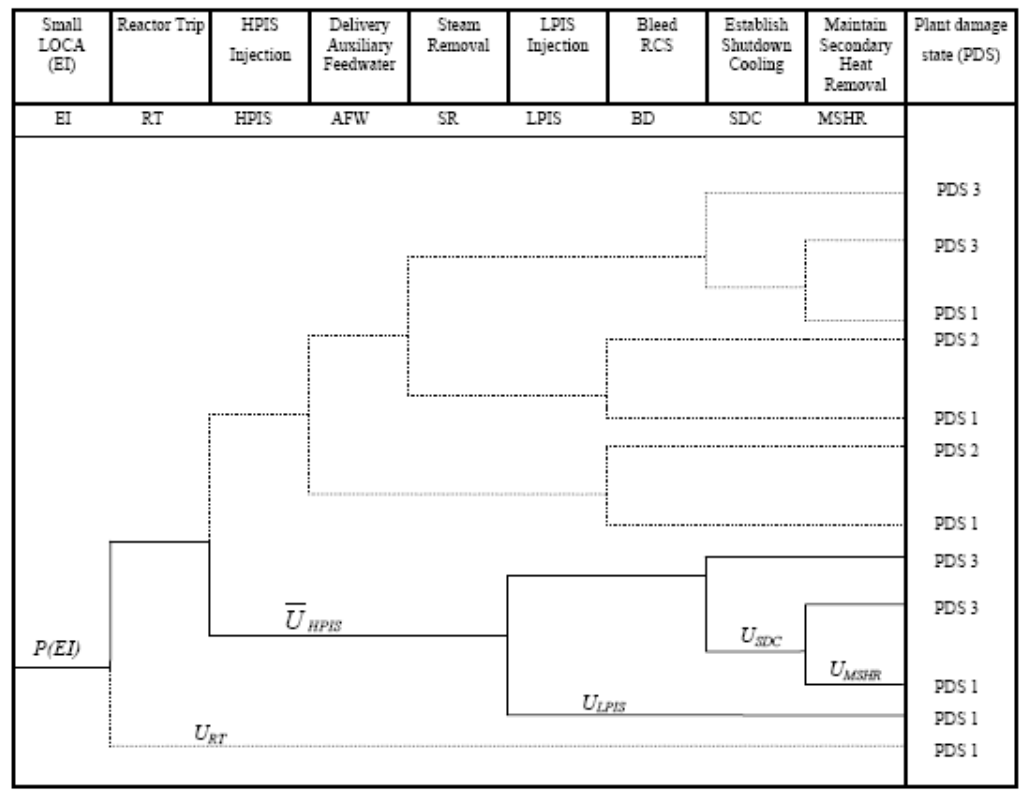

Figure 2: Small LOCA event tree ${ }^{17}$

The accident sequences considered for the quantification of the accident costs are those which involve the failure of the HPIS (thick lines in Error! Reference source not found.), so that the possible Plant Damage States (PDS) are PDS1 and PDS3. Thus: 
$\left\{\begin{array}{c}C_{\text {accident }}=C_{1}+C_{3} \\ C_{1}=P(E I) \cdot\left(1-U_{R T}\right) \cdot \bar{U}_{H P I S} \cdot\left\{U_{L P I S}+\left(1-U_{L P I S}\right) \cdot U_{S D C} \cdot U_{M S H R}\right\} \cdot C_{P D S 1} \\ C_{3}=P(E I) \cdot\left(1-U_{R T}\right) \cdot \bar{U}_{H P I S} \cdot\left(1-U_{L P I S}\right) \cdot\left\{\left(1-U_{M S H R}\right) \cdot U_{S D C}+\left(1-U_{S D C}\right)\right\} \cdot C_{P D S 3}\end{array}\right.$

where $C_{1}$ and $C_{3}$ are the total costs associated with accident sequences leading to damaging states 1 and 3 , respectively. These costs depend on the initiating event frequency $P(E I)$ and on the unavailability values $U_{i}$ of the safety systems which ought to intervene along the various sequences: these values are taken from the literature. ${ }^{13,17}$ Rates of Initiating Events at United States Nuclear Power Plants: 1987-1995) for all systems except for the SDC and MSHR, which were not available and were arbitrarily assumed of the same order of magnitude of the other safety systems, and for the HPIS for which the unavailability $\bar{U}_{\text {HPIS }}$ is calculated from (2) and (5) and it depends on the TIs of the components. Finally, for the values of $C_{P D S 1}$ and $C_{P D S 3}$, the accident costs for PDS1 and PDS3, respectively, are taken as the mean values of the uniform distributions given in Yang, Hwang, Sung and Jin. ${ }^{17}$ Table 2 summarizes the input data.

\begin{tabular}{|l|l|l|l|l|l|l|}
\hline $\begin{array}{l}P(E I) \\
\left(\mathrm{y}^{-1}\right)\end{array}$ & $\begin{array}{l}U_{R T} \\
\left(\mathrm{y}^{-1}\right)\end{array}$ & $\begin{array}{l}U_{L P I S} \\
\left(\mathrm{y}^{-1}\right)\end{array}$ & $\begin{array}{l}U_{S D C} \\
\left(\mathrm{y}^{-1}\right)\end{array}$ & $\begin{array}{l}U_{M S H R} \\
\left(\mathrm{y}^{-1}\right)\end{array}$ & $\begin{array}{l}C_{P D S 1} \\
(\$ \times \text { event })\end{array}$ & $\begin{array}{l}C_{P D S 2} \\
(\$ \times \text { event })\end{array}$ \\
\hline $2.43 \cdot 10^{-5}$ & $3.6 \cdot 10^{-5}$ & $9 \cdot 10^{-3}$ & $5 \cdot 10^{-3}$ & $5 \cdot 10^{-3}$ & $2.1765 \cdot 10^{9}$ & $1.375 \cdot 10^{8}$ \\
\hline \multicolumn{5}{|c|}{ Table 2: Accident cost input data } \\
\hline
\end{tabular}

the exposure time $E T$ due to the tests and possible maintenance activities on a single component $h$ can be computed as:

$E T_{h}(\theta)=\frac{T M}{\tau_{h}} t_{h}+\left(\rho_{h}+\lambda_{h} \tau_{h}\right) \frac{T M}{\tau_{h}} d_{h}, h=1, \ldots ., N_{c}$

Then,

$$
E T(\theta)=\sum_{h=1}^{N_{c}} E T_{h}(\theta)
$$

The multiobjective optimization problem (2)-(4) has been solved using the MOGA code developed at the Laboratorio di Analisi di Segnale e Analisi di Rischio (LASAR, Laboratory of Signal Analysis and Risk Analysis, http://lasar.cesnef.polimi.it/); the input parameters and settings are reported in Error! Reference source not found. ${ }^{9}$ 


\begin{tabular}{|l|l|}
\hline Number of chromosomes $\left(N_{p}\right)$ & 100 \\
\hline Number of generations (termination criterion) & 500 \\
\hline Selection & Standard Roulette \\
\hline Replacement & Random \\
\hline Mutation probability & $5 \cdot 10^{-3}$ \\
\hline Crossover probability & 1 \\
\hline Number of non-dominated solutions in the archive & 100 \\
\hline \multicolumn{2}{|c|}{ Table 3: MOGA input parameters and rules } \\
\end{tabular}

The resulting Pareto Set $(\Theta)$ is made of 100 points, and the corresponding Pareto Frontier is showed in Error! Reference source not found. in the objective functions space.

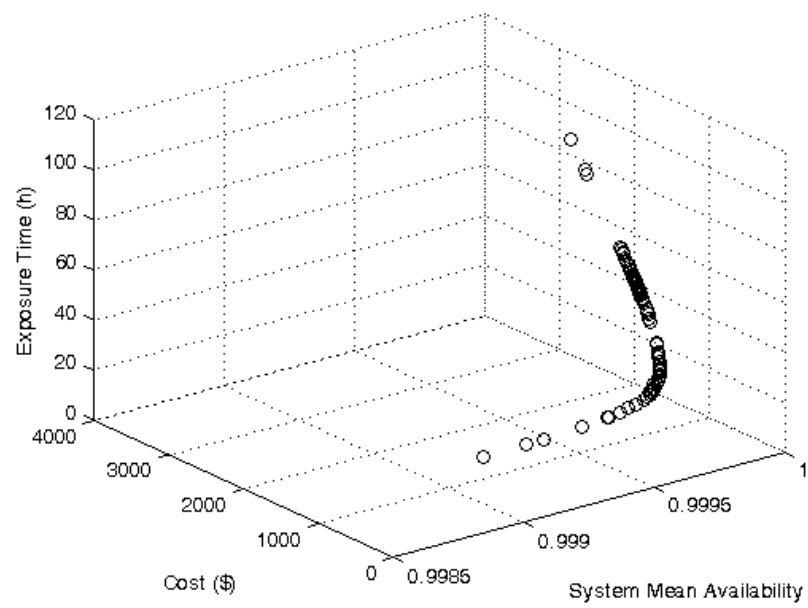

Figure 3 : Pareto Frontier, in the objective functions space, obtained by the MOGA code

\section{Decision support methods}

\subsection{A posteriori approaches}

When analyzing the Pareto Frontier, the DM either:

looks for the solution closest to the ideal one, i.e., that which optimizes all the objectives simultaneously;

applies his or her preferences on the objective functions values to identify the best solution according to these preferences.

The two decision situations, i.e., in presence or not of preferences on the objectives values, may lead to the selection of different solutions and 
require different procedures of reduction of the solutions in the Pareto Frontier. To this purpose, different a posteriori procedures can be developed to aid the DM in selecting the preferred solution; two of these are synthetically illustrated in the following.

The first a posteriori method presented below, is based on a two-step procedure developed by the authors, for which the availability of the software has rendered possible the comparison on a literature case study. The second method based on the Self Organizing Maps and Data Envelopment Analysis has been proposed elsewhere in the literature and its application is here critically evaluated.

\subsubsection{Subtractive clustering, fuzzy scoring and Level Diagrams for decision} making support ${ }^{20}$

A two-step procedure has been introduced by the authors in Zio and Bazzo. ${ }^{20}$ This procedure consists in grouping in "families" by subtractive clustering the non-dominated solutions of the Pareto Set, according to their geometric relative distance in the objective functions space (Pareto Frontier), and then selecting an "head of the family" representative solution within each cluster. Level Diagrams ${ }^{2}$ are used to effectively represent and analyze the reduced Pareto Frontiers; they account for the distance of the Pareto Frontier and Set solutions from the ideal (but not feasible) solution, optimal with respect to all the objectives simultaneously.

Considering a multiobjective problem with $l$ objectives to be minimized, $m$ to be maximized (such that $N_{o b j}=l+m$ ), $n$ solutions in the Pareto Set, and indicating by $\underline{J}\left(\theta^{i}\right)=\left(\begin{array}{lllll}J_{1}\left(\theta^{i}\right) & \ldots & J_{s}\left(\theta^{i}\right) & \ldots & J_{\text {Nobj }}\left(\theta^{i}\right)\end{array}\right)$ the objective functions values vector corresponding to the solution $\theta^{i}$ in the Pareto Set $\Theta, i=1, \ldots ., n$, the distance of each Pareto solution from the optimal solution can be measured in terms of the following 1-norm: 1-norm :

$\left\|\underline{J}\left(\theta^{i}\right)\right\|_{1}=\sum_{s=1}^{N o b j} J_{s, n o r m}\left(\theta^{i}\right)$, with $0 \leq\left\|\underline{J}\left(\theta^{i}\right)\right\|_{1} \leq s, s=1, \ldots ., N_{o b j}$ where each objective value $J_{s}\left(\theta^{i}\right)$, is normalized with respect to its minimum and maximum values $\left(J_{s}^{\min }\right.$ and $J_{s}^{\max }$ ) on the Pareto Frontier ${ }^{2}$ as follows: 
$J_{s, \text { norm }}\left(\theta^{i}\right)=\frac{J_{s}\left(\theta^{i}\right)-J_{s}^{\min }}{J_{s}^{\max }-J_{s}^{\min }} \quad s=1, \ldots ., l$

and

$J_{s, n o r m}\left(\theta^{i}\right)=\frac{J_{s}^{\max }-J_{s}\left(\theta^{i}\right)}{J_{s}^{\max }-J_{s}^{\min }} \quad s=1, \ldots ., m$

Subtractive clustering operates on the normalized objective values $\underline{J}_{n o r m}\left(\theta^{i}\right)$, $i=1, \ldots ., n$ and groups the non-dominated solutions in "families" according to their geometrical distance; it starts by calculating the following potential $P\left(\underline{J}_{\text {norm }}\left(\theta^{i}\right)\right):^{5}$

$P\left(\underline{J}_{\text {norm }}\left(\theta^{i}\right)\right)=\sum_{l=1}^{n} e^{-\alpha\left\|\underline{J}_{\text {norm }}\left(\theta^{i}\right) \underline{J}_{\text {norm }}\left(\theta^{l}\right)\right\|^{2}}, \alpha=\frac{4}{r_{a}^{2}}$

where $r_{a}$, the cluster radius, is a parameter which determines the number of clusters that will be identified. The first cluster center $J_{\text {norm }}^{1}$ is selected as the solution with the highest potential value $P\left(\underline{J}_{\text {norm }}^{1}\right)$. All the other $n-1$ solutions potentials $P\left(\underline{J}_{\text {norm }}\left(\theta^{4}\right)\right)$ are corrected subtracting the potential $P\left(\underline{J}_{\text {norm }}^{1}\right)$ multiplied by a factor which considers the distance between the $i-t h$ solution and the first cluster center:

$$
\begin{aligned}
& P\left(\underline{J}_{\text {norm }}\left(\theta^{i}\right)\right)=P\left(\underline{J}_{n o r m}\left(\theta^{i}\right)\right)-P\left(\underline{J}_{\text {norm }}^{1}\right) e^{-\beta \|\left.\underline{J}_{\text {norm }}\left(\theta^{i}\right) \underline{-}_{\text {norm }}^{1}\right|^{2}}, \\
& \beta=\frac{4}{r_{b}^{2}} \text { and } r_{b}=q r_{a}
\end{aligned}
$$

where $q$ is an input parameter called squash factor, which indicates the neighborhood with a measurable reduction of potential expressed as a fraction of the cluster radius and is here set equal to 1.25.

Generally, for the the $j-t$ th cluster center found $\underline{J}_{n o r m}^{j}, j=1, \ldots ., K$, the potentials are reduced as follows:

$$
P\left(\underline{J}_{\text {norm }}\left(\theta^{i}\right)\right)=P\left(\underline{J}_{\text {norm }}\left(\theta^{i}\right)\right)-P\left(\underline{J}_{\text {norm }}^{j}\right) e^{-\beta \| \underline{J}_{\text {norm }}\left(\theta^{i}\right)-\left.\underline{-}_{\text {norm }}^{j}\right|^{2}}
$$

The process of finding new cluster centers and reducing the potential is repeated until a stopping criterion is reached. ${ }^{5}$

The cluster radius $r_{a}$ is chosen to maximize the quality of the resulting Pareto Frontier partition measured in terms of the silhouette value; ${ }^{15,16}$ for any cluster partition of the Pareto Frontier, a global silhouette index, $G S$, is computed as follows: 
$G S=\frac{1}{K} \sum_{j=1}^{K} S_{j}$

where $S_{j}$ is the cluster silhouette of the $j$-th cluster $F^{j}$, a parameter measuring the heterogeneity and isolation properties of the cluster, ${ }^{15,16}$ computed as the average value of the silhouette widths $s(i)$ of its solutions, defined as:

$s(i)=\frac{b(i)-a(i)}{\max \{a(i), b(i)\}}, i=1, \ldots, n$

where, $n$ is the number of solutions in the Pareto Set, $a(i)$ is the average distance from the $i-t h$ solution of all the other solutions in the cluster, and $b(i)$ is the average distance from the $i-t h$ solution of all the solutions in the nearest neighbor cluster, containing the solutions of minimum average from the $i-t h$ solution, on average.

A head of the family must then be chosen as the best representative solution of each cluster. If no DM preferences are given, the solution with the lowest 1norm value in each cluster is chosen as the best representative solution; according to the Level Diagrams definition, this means that the selected solution is the closest to the ideal solution, optimal with respect to all objectives. If, on the other hand, the DM preferences on the objective values are available, the best solutions for the DM can be assigned classes of merit with respect to the DM preferences, by setting objective values thresholds. Let us consider the Pareto Set $\Theta$ made of $n$ solutions; to the $i$-th solution $\theta^{i}(i=1, \ldots, n)$ corresponds a vector of objective values

$$
\underline{J}\left(\theta^{i}\right)=\left(\begin{array}{llll}
J_{1}\left(\theta^{i}\right) & \left.J_{2}\left(\theta^{i}\right) \quad \ldots \quad J_{\text {Nobj }}\left(\theta^{i}\right)\right)
\end{array}\right.
$$

where $N_{o b j}$ is the number of objective functions of the optimization problem. The objective values thresholds are given in a preference matrix $\mathrm{P}\left(N_{o b j} \times C\right)$, where $\mathrm{C}$ is the number of objective functions thresholds used for the classification, defining $C+1$ preference classes as in Error! Reference source not found. ${ }^{2}$ 

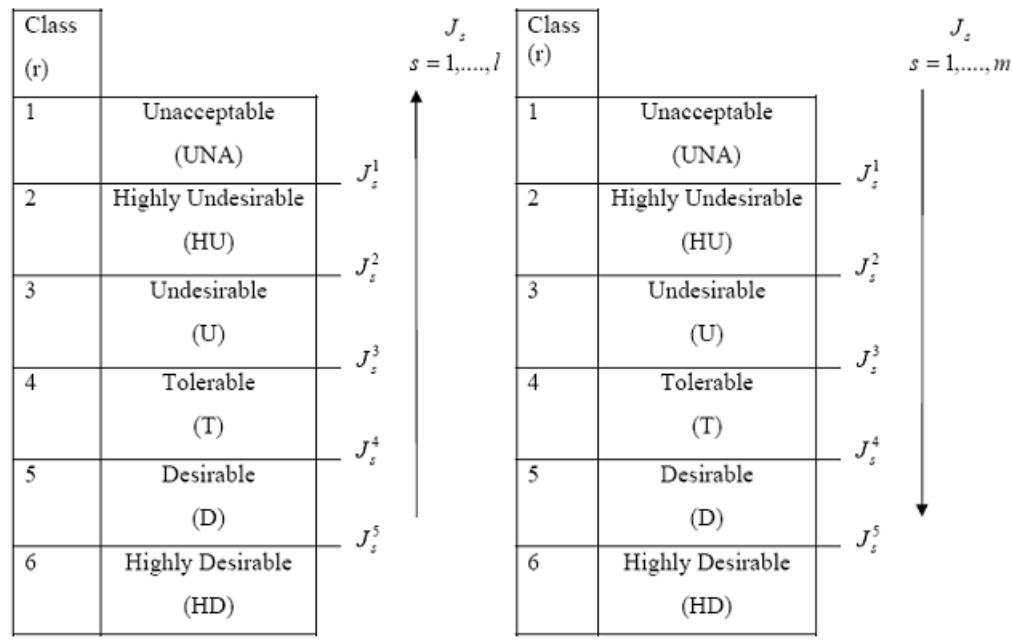

Figure 4: Class Thresholds assignment

where $J_{s}^{Z}, Z=1, \ldots, 5$ are the thresholds values of the $s$-th objective, $l$ and $m$ are the number of objectives to be minimized and maximized, respectively.

The fuzzy scoring procedure introduced by the authors in Zio and Bazzo ${ }^{19}$ is then applied: each preference class is assigned a score $s v(r),{ }^{2} r=1, \ldots ., C+1$, such that:

$s v(C+1)=0 ; s v(r)=N_{o b j} \cdot s v(r+1)+1$, for $r=C, \ldots, 1$

and each objective value $J_{s}\left(\theta^{i}\right), i=1, \ldots, n$ and $s=1, \ldots, N_{o b j}$, is assigned a membership function $\mu_{A^{r}}\left(J_{s}\left(\theta^{i}\right)\right)$ which represents the degree with which $J_{s}\left(\theta^{i}\right)$ is compatible with the fact of belonging to the $r$-th preference class, $r=1, \ldots ., C+1$.

A vector of $C+1=6$ membership functions is then defined for each objective $J_{s}$ :

$\underline{\mu}\left(J_{s}\left(\theta^{i}\right)\right)=\left(\mu_{A_{s}^{1}}^{s}\left(J_{s}\left(\theta^{i}\right)\right) \quad \mu_{A_{s}^{2}}\left(J_{s}\left(\theta^{i}\right)\right) \quad \mu_{A_{s}^{3}}\left(J_{s}\left(\theta^{i}\right)\right) \quad \mu_{A_{s}^{4}}\left(J_{s}\left(\theta^{i}\right)\right) \quad \mu_{A_{s}^{s}}\left(J_{s}\left(\theta^{i}\right)\right) \quad \mu_{A_{s}^{6}}\left(J_{s}\left(\theta^{i}\right)\right)\right)$ ,

$i=1, \ldots ., n, s=1, \ldots ., N_{o b j}$.

The membership-weighted score of each individual objective is then computed; given the scoring vector $\underline{s v}=\left(\begin{array}{llll}s v(1) & s v(2) & \ldots . & s v(C+1)\end{array}\right)$, whose components are defined in (19), and the membership functions vector $\mu\left(J_{s}\left(\theta^{i}\right)\right)$ in (20) for the $i-t h$ solution and $s$-th objective function, the score $s v_{s}^{i}$ of the individual objective $J_{s}$ is obtained by weighting the score $s v\left(r_{s}\right)$ of each class 
$r_{s}$ the objective belongs to, by the respective membership function value $\mu_{A_{s}^{r_{s}}}\left(J_{s}\left(\theta^{i}\right)\right), r_{s}=1, \ldots, 6$, and then summing the 6 resulting terms. This can be formulated in terms of the scalar product of the vectors $\mu\left(J_{s}^{i}\right)$ and $\underline{s v}$ as follows:

$$
s v_{s}^{i}=\frac{\left\langle\underline{\mu}\left(J_{s}\left(\theta^{i}\right)\right), \underline{s v}\right\rangle}{\sum_{r_{s}=1}^{6} \mu_{A_{s}^{r_{s}}}\left(J_{s}\left(\theta^{i}\right)\right)}, i=1, \ldots ., n \text { and } s=1, \ldots ., N_{o b j},
$$

where the denominator serves as the normalization factor.

Then, the score $S\left(\underline{J}\left(\theta^{i}\right)\right)$ of the $i-t h$ solution is the sum of the scores of the individual objectives

$$
S\left(\underline{J}\left(\theta^{i}\right)\right)=\sum_{s=1}^{N_{o b j}} s v_{s}^{i}, i=1, \ldots, n
$$

and the lowest score is taken as the most preferred solution.

According to this fuzzy scoring procedure, the head $\underline{H}^{j}$ of the generic family $F^{j}, j=1, \ldots ., K$, is chosen as the solution in $F^{j}$ with lowest scores $S\left(J\left(\theta^{i}\right)\right)$ : $S\left(\underline{H}^{j}\right)=\min S\left(\underline{J}\left(\theta^{i}\right)_{k}\right), k=1, \ldots ., n^{j}$ and $j=1, \ldots ., K$

Level Diagrams ${ }^{2}$ are finally used to represent and analyse the reduced Pareto Frontier thereby obtained.

With reference to the Pareto Frontier of Error! Reference source not found. for the test intervals optimization case study, the maximum value of the global silhouette (0.71) is found in correspondence of a cluster radius equal to 0.18 , as showed in Error! Reference source not found., which results in $K=9$ clusters. 


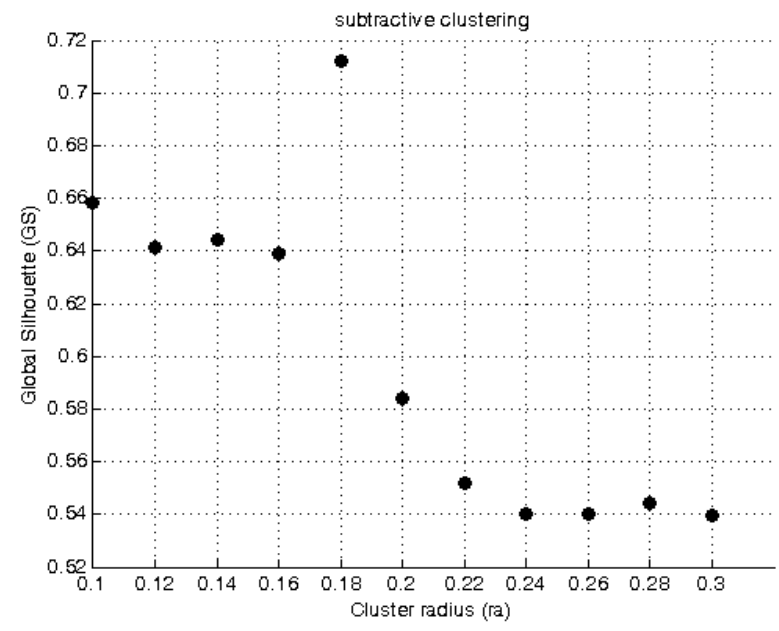

Figure 5: GS for different cluster radius values

For illustration purposes, let us introduce an arbitrary preference matrix $P$ for the test intervals optimization (Error! Reference source not found.).

\begin{tabular}{|c|c|c|c|c|c|}
\hline & $J_{s}^{1}$ & $J_{s}^{2}$ & $J_{s}^{3}$ & $J_{s}^{4}$ & $J_{s}^{5}$ \\
\hline$J_{1}$ & 0.9975 & 0.998 & 0.9985 & 0.999 & 0.9995 \\
\hline$J_{2}$ & 900 & 800 & 700 & 600 & 500 \\
\hline$J_{3}$ & 60 & 50 & 45 & 40 & 30 \\
\hline
\end{tabular}

Table 4: Preference threshold matrix $P$ 
The reduced Pareto Frontier is showed in Error! Reference source not found.: the best solutions (the dark circles) can be easily identified; there are also 4 solutions (the white circles) which have high score values, and thus are unacceptable, i.e., not interesting for the DM.

Note that for the application of the method, the DM only has to select the optimum cluster radius (from Error! Reference source not found.), define the preference matrix and use the Level Diagrams representation to evaluate the solutions according to their distance from the ideal solution, optimal with respect to all objectives.
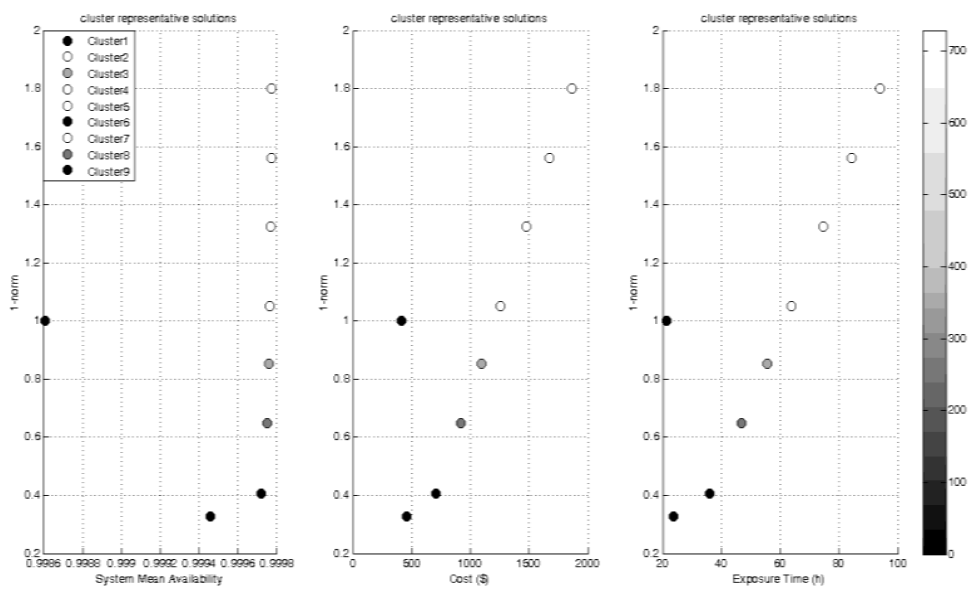

Figure 6: Level Diagrams representation of the family representative solutions with lowest score $S\left(H^{j}\right)$

3.1.2 Self-Organizing Maps solution clustering and Data Envelopment Analysis solution pruning for decision making support ${ }^{I I}$

Another approach to simplifying the decision making in multiobjective optimization problems has been introduced in Li, Liao and Coit, ${ }^{11}$ based on Self Organizing Maps (SOM) ${ }^{8}$ and Data Envelopment Analysis (DEA) ${ }^{7} 7$.

The Pareto optimal solutions are first classified into several clusters by applying the SOM method, an unsupervised classification method based on a particular artificial neural network with a single layer feedforward structure. Then, non-efficient solutions are eliminated from each cluster and representative efficient solutions are identified, by application of the data envelopment analysis (DEA) method which is a particular multiobjective selection optimization 
approach. For the efficiency selection, DEA considers an indicator of input/output solution performance based on a predefined relative efficiency criterion: in a multiobjective problem, some objectives can be considered as inputs, e.g., cost, exposure time, which typically have to be minimized, and others can be considered as outputs, e.g., availability, profits, which have to be maximized. Let us consider a problem with $l$ inputs and $m$ outputs; then, for the $i$ th solution $\theta^{i}(i=1, \ldots, n)$ in the Pareto Set $\Theta$ one can define a relative efficiency as :

$$
R E\left(\theta^{i}\right)=\frac{\text { weighted sum of outputs }}{\text { weighted sum of inputs }}=\frac{\sum_{k=1}^{m} x_{i, k} J_{k}\left(\theta^{i}\right)}{\sum_{h=1}^{l} v_{i, h} J_{m+h}\left(\theta^{i}\right)}
$$

where $J_{k}\left(\theta^{i}\right), k=1, \ldots ., m$, are the outputs, i.e., the objectives to be maximized, $J_{m+h}\left(\theta^{i}\right), h=1, \ldots ., l$, are the inputs, i.e., the objectives to be minimized, $v_{i, h}$ and $x_{i, k}$ are the weights of the inputs and outputs, respectively. The problem of computing the $R E\left(\theta^{i}\right)$ values is framed as a particular multiobjective problem for each solution, where the weights are the decision variables and the relative efficiency is the objective function to be maximized:

$$
\max _{u_{i, k}, v_{i, h}} R E\left(\theta^{i}\right)=\max _{u_{i, k}, v_{i, h}} \frac{\sum_{k=1}^{m} x_{i, k} J_{k}\left(\theta^{i}\right)}{\sum_{h=1}^{l} v_{i, h} J_{m+h}\left(\theta^{i}\right)}
$$

The Pareto Frontier is then reduced to the solutions with the highest relative efficiency values $R E\left(\theta^{i}\right)$ and the DM is provided with a small number of most efficient solutions.

This method has been showed to be effective in reducing the number of possible solutions to be presented to the DM in a multiobjective reliability allocation problem, ${ }^{11}$ but not with the inclusion of the DM preferences. The solution selection is based only on a solution performance criterion (the relative efficiency), but in presence of particular requirements on the objective values, the solutions most preferred by the DM might not be the most efficient ones. Also, the DEA method solves a maximization problem for each solution and this increases the computational time, particularly for large Pareto Frontiers. 


\subsection{A priori approach}

The a priori approach considered in this work is the Guided Multiobjective Genetic Algorithm (G-MOGA). ${ }^{18}$ The deep knowledge of this method codeveloped by one of the authors, makes it a suitable a priori method for detailed comparison on the literature case study.

DM preferences are taken into account by modifying the definition of dominance used for the multiobjective optimization. ${ }^{3,4}$ In general, dominance is determined by pair-wise vector comparisons of the multiobjective values corresponding to the pair of solutions under comparison; specifically, solution $\theta^{1}$ dominates solution $\theta^{2}$ if

$\forall i \in\{1, \ldots, s\}, J_{i}\left(\theta^{1}\right) \leq J_{i}\left(\theta^{2}\right) \wedge \exists k \in\{1, \ldots, s\}: J_{k}\left(\theta^{1}\right)<J_{k}\left(\theta^{2}\right)$.

The G-MOGA is based on the idea that the DM is able to provide reasonable trade-offs for each pair of objectives.

For each objective, a weighted utility function of the objective vector $\underline{J}\left(\theta^{i}\right)=\left(\begin{array}{lllll}J_{1}\left(\theta^{i}\right) & \ldots & J_{s}\left(\theta^{i}\right) & \ldots & \left.J_{\text {Nobj }}\left(\theta^{i}\right)\right)\end{array}\right)$ is defined as follows:

$\Omega_{s}\left(\underline{J}\left(\theta^{i}\right)\right)=J_{s}\left(\theta^{i}\right)+\sum_{s=1, p \neq s}^{N_{o b j}} a_{s p} \cdot J_{p}\left(\theta^{i}\right), i=1, \ldots ., n$ and $s=1, \ldots ., N_{o b j}$

where the coefficients $a_{s p}$ indicate the amount of loss in the $s$-th objective that the DM is willing to accept for a gain of one unit in the $p$-th objective, $s, p=1, \ldots ., N_{o b j}$ and $p \neq s$. Obviously $a_{s s}=1$. The domination definition is then modified as follows with reference to a minimization problem, for example: $\theta^{1}$ dominates another solution $\theta^{2}$ if

$\forall i \in\{1, \ldots ., s\}, \Omega_{i}\left(\underline{J}\left(\theta^{1}\right)\right) \leq \Omega_{i}\left(\underline{J}\left(\theta^{2}\right)\right) \wedge \exists k \in\{1, \ldots ., s\}: \Omega_{k}\left(\underline{J}\left(\theta^{1}\right)\right)<\Omega_{k}\left(\underline{J}\left(\theta^{2}\right)\right)$

The guided domination allows the DM to change the shape of the dominance region and to obtain a Pareto Frontier focused on the preferred region, defined by the maximally acceptable trade-offs for each pair of objectives.

The G-MOGA developed at LASAR has been applied to the test interval optimization case study of Section 1.0 and the $a_{s p}$ coefficients are given in Error! Reference source not found..

\begin{tabular}{|l|l|}
\hline Preference & G-MOGA trade-offs $\left(a_{s p}\right)$ \\
\hline$J_{1}$ much less important than $J_{2}$ & $a_{12}=5, a_{21}=0$ \\
\hline$J_{1}$ much less important than $J_{3}$ & $a_{13}=100, a_{31}=0$ \\
\hline$J_{2}$ more important than $J_{3}$ & $a_{23}=10, a_{32}=0.1$ \\
\hline
\end{tabular}


Table 5: $a_{s p}$ coefficients for the test intervals optimization case study

To obtain results comparable to those of the a posteriori preference assignment, the a priori preferences in the first column of Error! Reference source not found. have been set considering the threshold values assigned in the preference matrix $P$ of Error! Reference source not found.. Since the system mean availability unacceptable threshold value $\left(J_{1}^{1}\right)$ is below the minimum value of the objective in the Pareto Frontier (0.9986), i.e., all the results are at least acceptable, the system mean availability is considered as the least important objective, and thus $a_{21}$ and $a_{31}$, which indicate the amounts of loss in the cost and exposure time objectives, respectively, that the DM is willing to accept for a gain of one unit in the system mean availability objective, are both set to 0 . The cost and the workers' exposure time unacceptable threshold values ( $900 \$$ and 60 h respectively, Error! Reference source not found.) are inside the objective values ranges in the Pareto Frontier ([416.23,2023] and [21.42,102]). In particular, considering the unacceptable thresholds values normalized by the objective range width

$\overline{J_{s}^{1}}=\frac{J_{s}^{1}}{\max _{i}\left(J_{s}\left(\theta^{i}\right)\right)-\min _{i}\left(J_{s}\left(\theta^{i}\right)\right)}$,

for these two objectives to be maximized the results are $\overline{J_{2}^{1}}=0.56$ and $J_{3}^{1}=0.75$, which indicate that the cost objective presents the strongest restrictions on the objective values, because the unacceptable threshold value is closer to the cost minimum value. For this reason, cost is considered a more important objective than the worker's exposure time. To transform these linguistic preferences into numerical values for the $a_{s p}$ coefficients, $s, p=1, \ldots . ., N_{o b j}$ and $p \neq s$, the degradation of the objective $J_{s}\left(\Delta^{-}\left(J_{s}\right)\right.$, in physical units) equivalent to an increment in the objective $J_{p}\left(\Delta^{+}\left(J_{p}\right)\right.$, in physical units) has to be computed; the $a_{s p}$ coefficients can be found as:

$a_{s p}=\frac{\Delta^{-}\left(J_{s}\right)}{\Delta^{+}\left(J_{p}\right)}$

The other G-MOGA settings are the same as those of the standard MOGA applied in Section 1.0. 


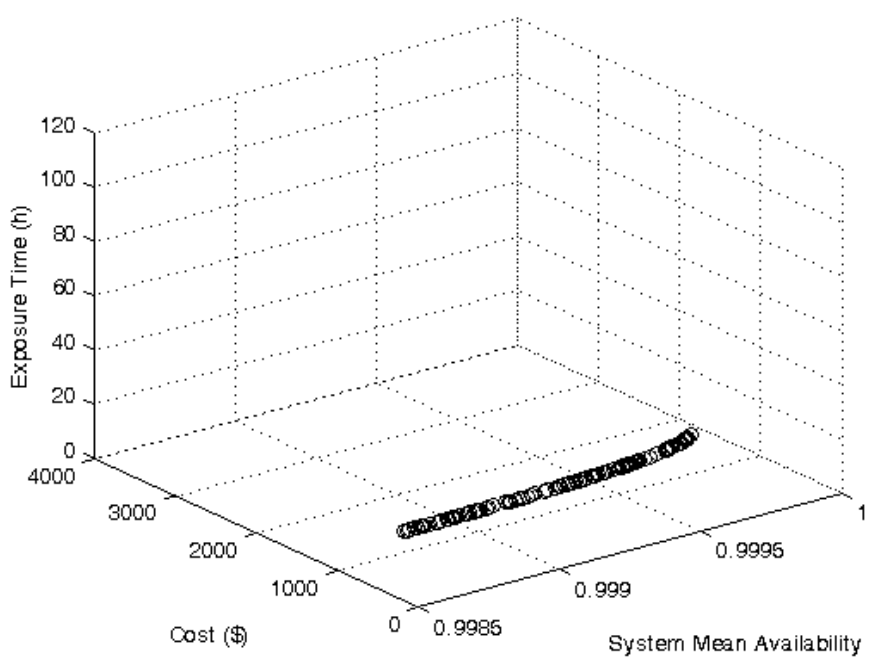

Figure 7: Pareto Frontier obtained with the G-MOGA algorithm

The Pareto Frontier obtained with the G-MOGA (Error! Reference source not found.) is a section of the original Pareto Frontier of Error! Reference source not found., whose solutions are characterized by low cost and exposure time values. Note that the ranges of these two latter objectives are significantly reduced $([402.98,497.06]$ and $[20.74,25.651]$, respectively), while the range of the system mean availability $([0.9986,0.996])$ is approximately the same; this is due to the lower importance given to the system availability objective.

The Pareto Frontier is dense (still made of 100 solutions) but concentrated in the preferred region of the objective functions space: this means that the algorithm is capable of finding a number of solutions which are preferred according to the DM requirements. This increases the efficiency of the solutions offered to the DM but the decision problem is still difficult because the DM has to choose between very close preferred solutions.

The procedure of solution reduction by clustering illustrated in Section 1.0 could, then, be applied to the concentrated, preferred Pareto Frontier. In this case, given the narrow objective values ranges, particularly for the cost and the worker's exposure time objectives, it may be difficult to assign preferences on the objectives values. For this reason, the selection of the best representative solution is performed in absence of preferences on the objectives values (Section 1.0). The optimal cluster radius $\left(r_{a}\right)$ which maximizes the global silhouette value is equal to 0.32 , which corresponds to a number of clusters $K=5$. 


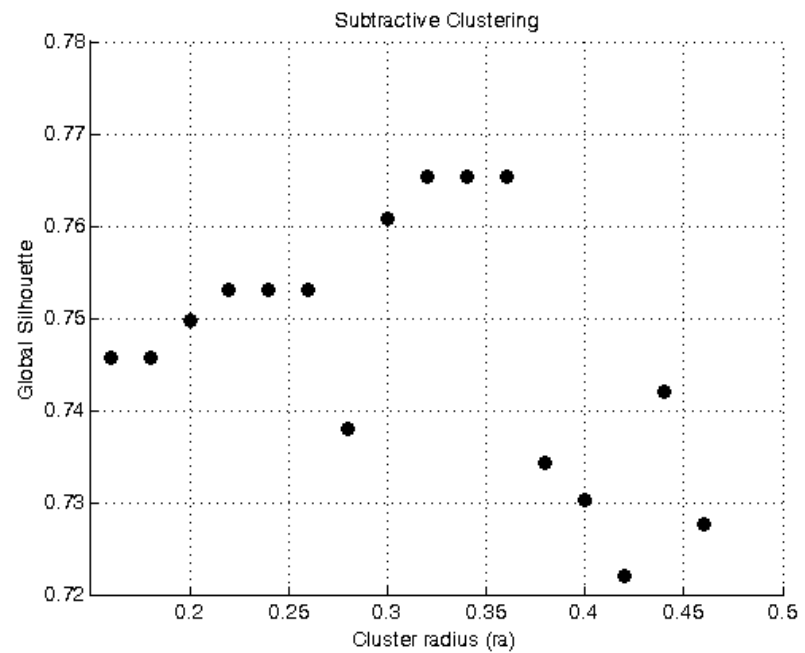

Figure 8: GS for different cluster radius values

The resulting cluster representative solutions, i.e., the solutions in each cluster closest to the optimal point, ideal with respect to all the objectives are showed by Level Diagrams in Error! Reference source not found.
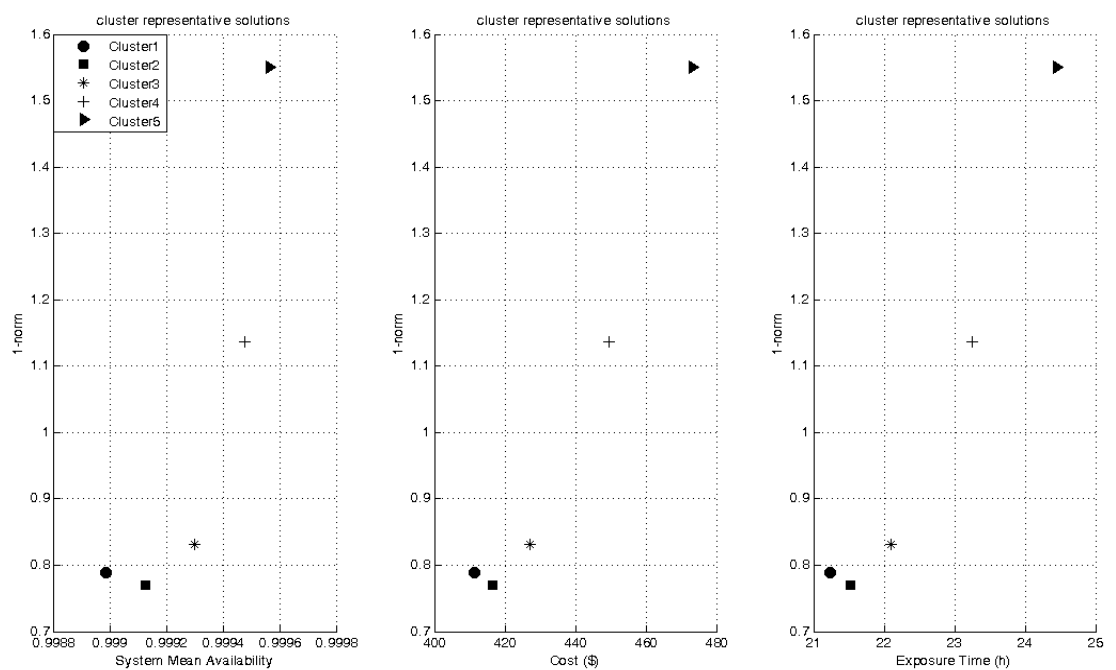

Figure 9: Level Diagrams representation of the family representative solutions closest to the ideal solution optimizing all objectives 
Given the regular and concentrated Pareto Frontier obtained with the GMOGA algorithm, the optimal number of clusters, and thus of representative solutions, is smaller than in the previous case; the combined application of the GMOGA algorithm and clustering procedure is found to provide a small number of preferred solutions, which make it easier for the DM to choose the final solution: the clustering procedure is really effective in reducing the number of solutions to be presented to the DM, overcoming the problem of the crowded Pareto Frontier made of close solutions in the preferred region of the domain.

On the other hand, to compute the $a_{s p}$ coefficients to introduce DM's reasonable trade-offs, one has to know the expressions of the objective functions as implemented in the search algorithm, since, for computational reasons, these expressions might be different from those of the problem statement, e.g., to enhance the procedure of maximization or minimization. Then, if the DM is not satisfied with the resulting Pareto Frontier, he or she has to modify the input parameters of the genetic algorithm. These requests to the DM might be excessive in practical applications because, as showed before, to compute the trade-offs coefficients the DM must, at least, know the orders of magnitude of the objectives. Without any reference value it would be then complicated to define the amount of an objective that the DM accepts to give up for a unitary increase of another objective. Moreover, this task becomes particularly burdensome for problems with more than two objectives, as the required number of trade-offs to be specified increases dramatically with the number of objectives. ${ }^{18}$

\section{Conclusions}

The results of algorithms of multiobjective optimization amount to a Pareto Set of non-dominated solutions among which the DM has to select the preferred ones. The selection is difficult because the set of non-dominated solutions is usually large, and the corresponding representative Pareto Frontier in the objective function space crowded.

In the end, the application of DM preferences drives the search of the optimal solution and can be done mainly a priori or a posteriori.

In this work, a comparison of some a priori and a posteriori methods of preference assignment is proposed. The methods have been chosen because the authors have the depth of experience on them necessary for a detailed comparison, here performed on a case study concerning the optimization of the 
test intervals of the components of a nuclear power plant safety system. The a priori G-MOGA method considered has been showed to lead to a focalized Pareto Frontier, since the DM preferences are embedded in the genetic algorithm to bias the search for non-dominated solutions towards the preferred region; the a posteriori methods considered, on the other hand, have been showed effective in reducing the number of solutions on the Pareto Frontier.

From the results of the comparative analysis, it turns out that the a priori and a posteriori approaches considered are not necessarily in contrast but can be combined to obtain a reduced number of optimal solutions focalized in a preferred region, to be presented to the DM for the decision.

However, the implementation of the a priori method seems more complicated because it requires the assignment of preference trade-offs on the objectives values; this latter task is difficult if the DM has no experience on the specific multiobjective problem, and the complexity increases with the number of the objectives. In these cases, a posteriori procedures can be applied alone, still with satisfactory results. In particular, the two-steps clustering procedure introduced by the authors for identifying a small number of representative solutions to be presented to the DM for the decision, has been showed to be an effective tool which can be applied in different decision situations independently of the Pareto Frontier size and the number of objective functions.

\section{References}

1. ATKOSoft, Survey of Visualization Methods and Software Tools, (1997).

2. X. Blasco, J.M. Herrero and J. Sanchis, M. Martínez, Multiobject. Optim., Inf. Sci., 178, 39083924 (2008).

3. J. Branke, T. Kaubler and H. Schmeck, Adv. Eng. Software, 32, 499 (2001).

4. J. Branke, T. Kaubler and H. Schmeck, Tech. Rep. TR no.399, Institute AIFB, University of Karlsruhe,Germany (2000).

5. S. Chiu, J. of Intell. \& Fuzzy Syst., 2 (3), 1240 (1994).

6. C.A. Coello Coello, C, in 2000 Congress on Evolut. Comput. (IEEE Service Center, Piscataway NJ, 2000), Vol.1, p. 30.

7. WW. Cooper, L.M. Seiford and K. Tone, Data Envelopment Analysis: a Comprehensive Text with Models, Applications, References, and DEA-Solver Software (Springer, Berlin, 2006).

8. L. Fausett, Fundamentals of Neural Networks: Architectures, Algorithms, and Applications (Prentice-Hall, Englewood Cliffs, 1994).

9. P. Giuggioli Busacca, M. Marseguerra and E. Zio, Reliab. Eng. Syst. Saf., 72, 59 (2001).

10. ICRP Publication 60, Annals of the ICRP, 21, 1 (1991).

11. Z. Li, H. Liao and D.W. Coit, Reliab. Eng. Syst. Saf., 94, 1585 (2009).

12. S. Martorell, S. Carlos, A. Sanchez and V. Serradell, Reliab. Eng. Syst. Saf., 67, 215 (2000). 
13. US Nuclear Regulatory Commission, Rates of Initiating Events at United States Nuclear Power Plants: 1987-1995, NUREG/CR- 5750 (1999).

14. L. Rachmawati and D. Srinivasan, in 2006 Congress on Evolut. Comput. (IEEE Conference Pubblications, 2006), p. 962-968.

15. P.J. Rousseeuw, J. Comput. Appl. Math., 20, 53 (1987).

16. P. Rousseeuw, E. Trauwaert and L. Kaufman, Belgian J. of Oper. Res., Stat. and Comput. Sci., 29(3), 35 (1989)

17. J.E. Yang, M.J. Hwang, T.Y. Sung and Y. Jin, Reliab. Eng. Syst. Saf., 65, 229 (1999).

18. E. Zio, P. Baraldi and N. Pedroni, Reliab. Eng. Syst. Saf., 94, 432 (2009).

19. E. Zio and R. Bazzo, Submitted to Inf. Sci. (2009).

20. E. Zio and R. Bazzo, Eur. J. of Oper. Res., 210(3), 624 (2011). 